\title{
Evidence-based infection control - Vom Nachweis der Effektivität infektionspräventiver Maßnahmen
}

\section{Roland Schulze-Röbbecke}

Aus gutem Grunde ist es in der wissenschaftlich begründeten Medizin üblich, vorzugsweise jene therapeutischen oder diagnostischen Maßnahmen anzuwenden, deren Effektivität mittels empirisch gewonnener Daten belegt ist. Gemäß den Grundsätzen der Evidence-based Medicine (EBM) [1] ist der beste empirische Beleg und höchste Erkenntniswert von klinischen Studien und deren Metaanalysen zu erwarten. Den verschiedenen Arten klinischer Studien (z. B. randomisierte, kontrollierte Studien, Fall-Kontroll-Studien, Interventionsstudien) kommt hierbei eine unterschiedliche Aussagekraft zu. Neben klinischen Studien stehen weitere wissenschaftliche Erkenntnisquellen zur Verfügung, die allerdings mit einem höheren Risiko für Trugschlüsse einhergehen. Zu nennen sind hier insbesondere Berichte über Fallserien oder Einzelfälle sowie Experimente (z. B. in vitro oder Tierexperimente). Von niedrigster Aussagekraft ist dagegen die reine Expertenmeinung, die allein auf subjektiver Erfahrung und Einschätzung beruht und nicht auf wissenschaftlichen Daten.

Es kursieren Einwände, dass die Grundsätze der EBM und die Vorrangstellung der klinischen Studien zwar für die klinische Medizin gelten, nicht aber für die Hygiene und Infektionsprävention.

1. Einwand: In der Primärprävention, also der eigentlichen Verhinderung der Krankheitsentstehung, würden sich klinische Studien verbieten, denn diese liefen auf die Beobachtung der Häufigkeit von Erkrankungsund Todesfällen hinaus, und solche Fälle gelte es ja gerade zu verhindern. Ziel der Primärprävention könne es nicht sein, Erkrankungen geschehen zu lassen, um anschließend präventive Schlussfolgerungen aus den Beobachtungen zu ziehen.

2. Einwand: Für Studien zum Beleg der Wirksamkeit hygienischer Maßnahmen seien solch hohe Fallzahlen erforderlich, dass sie nicht realisierbar sind.
3. Einwand: Viele hygienische Empfehlungen seien so evident, dass sie keines empirischen Effektivitätsnachweises bedürfen. Auch zum Nachweis der Wirksamkeit des Fallschirms beim Absprung aus einem Flugzeug seien keine randomisierten, kontrollierten Studien durchgeführt worden [2].

Diese und andere Einwände halten einer näheren Überprüfung nicht stand:

- Ignaz Semmelweis Untersuchung über die Händedesinfektion zur Prävention des Kindbettfiebers in den 1840er-Jahren zählt weltweit zu den ersten klinischen Studien überhaupt und hatte die Primärprävention nosokomialer Infektionen (NI) zum Gegenstand. Der Interventionseffekt dieser Studie war so dramatisch, dass es unethisch wäre, ihn mit einer randomisierten, kontrollierten Studie zu bestätigen. Seitdem wurde eine große Zahl klinischer Studien bis hin zu randomisierten, kontrollierten Studien publiziert, die sich mit der Primärprävention von Infektionen (einschließlich NI) befassen. Zitate finden sich z.B. in den einschlägigen evidenzbasierten Empfehlungen des Robert Koch-Instituts der letzten Jahre.

- Im Verlauf der Semmelweis-Studie starben zahlreiche Mütter. Entgegen dem ersten Einwand war die Durchführung aber weder unethisch noch unwissenschaftlich, denn ohne die Beobachtung von Morbiditäts- und Mortalitätszahlen hätte die Effektivität der Händedesinfektion nicht nachgewiesen werden können. Der damals vorherrschende Glaube an die überkommene Miasmentheorie oder an einen „Genius epidemicus“ wäre länger aufrechterhalten worden und es wären letztlich erheblich mehr Mütter gestorben.

- Auch der zweite Einwand ist nicht stichhaltig. Die Semmelweis-Studie und zahlreiche spätere Studien zur Prävention von NI wurden mit leicht verfügbaren und leicht handhabbaren Fallzahlen realisiert. Effekte, die sich nur mithilfe extrem hoher Fallzahlen nachweisen lassen, sind oft so gering, dass sie 
praktisch bedeutungslos sind. Das gilt sowohl für die klinische als auch für die Präventivmedizin.

- Dem heutigen Gebrauch von Fallschirmen gingen zahlreiche Experimente und Fall-Beobachtungen mit solch klarem Ergebnis voraus, dass randomisierte, kontrollierte Studien ethisch nicht vertretbar sind. Zudem sind die Funktionsweise eines Fallschirms und die ihr zugrunde liegenden physikalischen Gesetze leicht nachvollziehbar. Die meisten biologischen Vorgänge einschließlich der Infektionsübertragung folgen dagegen komplexen, nur schwer durchschaubaren Gesetzmäßigkeiten. Entscheidende Vorgänge spielen sich im mikroskopischen und submikroskopischen Bereich ab, der der direkten Beobachtung nicht zugänglich ist. So mussten etliche Hypothesen über Transmissionsmechanismen und Empfehlungen zur Prävention von NI, die gemäß dem dritten Einwand zunächst „evident“ erschienen, später revidiert werden, da sie falschen oder zu grob vereinfachenden Gedankenmodellen entsprangen und sich durch praktische Beobachtungen nicht belegen ließen. Als Beispiele sind der Einsatz von UV-Strahlen in OPs oder von Lüftungsanlagen mit Schwebstofffilterung in Intensivstationen zu nennen. Hätte Semmelweis den dritten Einwand gelten lassen und die Meinung der medizinischen Autoritäten seiner Zeit nicht angezweifelt, so hätte er allenfalls weitere Maßnahmen gegen Miasmen ergreifen, nicht aber einer neuen Hypothese nachgehen dürfen.

Besonders der dritte Einwand wurde und wird gerne dazu herangezogen, Infektionsprävention und Krankenhaushygiene nicht auf wissenschaftlicher Basis, sondern auf der Grundlage von Expertenmeinungen („eminenzbasiert“ [3]) zu praktizieren. Wie das folgende Beispiel zeigt, kann dies erhebliche finanzielle Folgen haben:

- Im Jahre 1990 wurde von KrankenhaushygieneExperten die rein „eminenzbasierte“ Empfehlung veröffentlicht [4], jedem Operationsraum als Nebenräume jeweils einen Einleitungsraum, einen Ausleitungsraum und einen Waschraum zuzuordnen (ein gemeinsamer Wasch- oder Ausleitungsraum für jeweils zwei benachbarte Operationsräume war jedoch „zulässig“). Raumlufttechnische (Klima-) Anlagen waren nach dieser Empfehlung gemäß DIN 1946 auszuführen, d.h. mit einer aufwendigen dreistufigen Filterung der Zuluft aller Räume der OP-Abteilung. Nach diesen Vorgaben wurden vor dem Jahr 2000 zahlreiche OP-Abteilungen konzipiert und gebaut.
- Zehn Jahre später revidierte dasselbe Expertengremium diese Empfehlung [5] und stellte fest, dass es ausreicht, für Narkoseeinleitung, Narkoseausleitung und Händewaschung in einer OP-Abteilung für mehrere Operationsräume nur noch jeweils eine Fläche oder einen Raum zur Verfügung zu stellen. Sofern eine raumlufttechnische Anlage installiert ist oder wird, braucht (abweichend von der DIN 1946, Teil IV) eine dreistufige Luftfilterung nur noch für die Operationsräume vorgesehen werden.

Dieser Wandel der Expertenmeinung brachte Einsparungen mit sich, die beim Neubau einer OP-Abteilung mit vier Operationsräumen bei etwa 344000 Euro liegen. Hinzu kommen deutlich niedrigere Betriebskosten durch den verringerten Belüftungsaufwand. Der Meinungswandel beruhte nicht auf neuen wissenschaftlichen Erkenntnissen aus dem Bereich der Infektionsprävention, sondern auf einem neuen „Bauchgefühl“, und führte zu keiner messbaren Erhöhung des Infektionsrisikos.

Dieses Beispiel zeigt einerseits einen begrüßenswerten Trend auf, andererseits zeigt es, auf welch tönernen Füßen rein „eminenzbasierte“ Empfehlungen stehen. Es wirft daher auch die Frage auf, mit welcher Berechtigung diese in vorgeblich wissenschaftlich begründeten Empfehlungen „nachdrücklich empfohlen“ werden dürfen (z.B. als Kategorie IB in den RKI-Empfehlungen [5]). Darüber hinaus stellt sich die Frage, wer für den wirtschaftlichen Schaden aufkommt, wenn Expertenmeinung ohne empirischen Beleg zur verbindlichen Richtschnur erhoben wird und sich im Nachhinein als gegenstandslos herausstellt.

Tatsächlich erscheinen „eminenzbasierte“ Forderungen teilweise in offiziellen, als „wissenschaftlich begründet“ deklarierten Empfehlungen der Krankenhaushygiene und in den Regelwerken des Arbeitsschutzes. Dort werden sie apodiktisch als „Stand des Wissens“ [6] bzw. als „Stand von Wissenschaft, Technik und Medizin“ [7] bezeichnet. Schlimmer noch: Wer von einer solch unzureichend begründeten Empfehlung abweichen will, kann von den Aufsichtsbehörden u. U. aufgefordert werden, die Ineffektivität der Empfehlung oder die Gleichwertigkeit eines alternativen Verfahrens nachzuweisen. Aus wissenschaftlicher und ethischer Sicht ist diese Vorgehensweise inakzeptabel. Sie hemmt den wissenschaftlichen Fortschritt und geht mit dem Risiko einher, Ressourcen für ineffektive Maßnahmen zu verschwenden. 
Es gibt kein vernünftiges Argument, welches der Infektionsprävention innerhalb der Medizin einen Sonderstatus hinsichtlich der Notwendigkeit des wissenschaftlichen Effektivitätsnachweises im Sinne der EBM einräumen würde. Wie in der klinischen Medizin haben auch in der Präventivmedizin die Experten und Fachgremien den Effektivitätsnachweis ihrer Empfehlungen zu erbringen. Den Kritikern „eminenzbasierter“ Empfehlungen gewissermaßen eine „Beweislastumkehr“ - also den Nachweis der Ineffektivität - aufzubürden, überträgt ihnen eine Aufgabe, die die Autoren der Empfehlungen nicht erfüllen konnten oder der sie sich entzogen haben.

Die reine Expertenmeinung ohne Effektivitätsnachweis als unumstößliches Dogma hinzustellen, hat in der Hygiene als medizinischem Fachgebiet eine gewisse Tradition und wird durch den Verzicht auf eine klare Definition des Begriffs „Hygiene“ [8] gefördert. Die Hygiene läuft dadurch Gefahr, sich aus der wissenschaftlich begründeten Medizin herauszulösen und Lehrinhalte zu vermitteln, die nur für „Eingeweihte“ nachvollziehbar sind. Für „Hygieneexperten“ ist die Versuchung groß, sich dieser Tendenz anzuschließen, wenn weder von wissenschaftlicher noch von behördlicher Seite Einspruch erhoben wird. Diejenigen aber, die Infektionsprävention ernst nehmen, sind aufgefordert, an krankenhaushygienische Empfehlungen und Leitlinien die gleichen wissenschaftlichen Qualitätsansprüche zu stellen, wie sie in der klinischen Medizin üblich sind. Die Einforderung des wissenschaftlichen Effektivitätsnachweises krankenhaushygienischer Empfehlungen ist umso zwingender, je teurer und aufwendiger die empfohlene Maßnahme ist bzw. je mehr sie die betroffenen Patienten unerwünschten Nebenwirkungen aussetzt. Denn letztendlich entscheidet das Verhältnis zwischen Effizienz und Kosten über den Erfolg der Empfehlungen und damit über die künftig dringend benötigte Akzeptanz der „Hygiene“ im praktischen Alltag.

\section{Literatur}

1 Sackett DL, Straus SE, Richardson WS, Rosenberg W, Haynes RB. Evidence-Based Medicine: How to Practice and Teach EBM. London: Churchill Livingstone, 2000

2 Smith GCS, Pell JP. Parachute use to prevent death and major trauma related to gravitational challenge: systematic review of randomised controlled trials. British Medical Journal 2003; 327: 14591461

3 Isaacs D, Fitzgerald D. Seven alternatives to evidence based medicine. British Medical Journal 1999; 319: 1618

4 Kommission für Krankenhaushygiene und Infektionsprävention des Bundesgesundheitsamtes. Anforderungen der Hygiene an die funktionell-bauliche Gestaltung von Operationsabteilungen, von Einheiten für kleine operative Eingriffe sowie von Untersuchungs- und Behandlungsräumen für operative Fachgebiete. Anlage zu Ziffer 4.3.3 der „Richtlinie für Krankenhaushygiene und Infektionsprävention“. Bundesgesundheitsblatt 1990; 33 : $270-272$

5 Kommission für Krankenhaushygiene und Infektionsprävention am Robert Koch-Institut. Anforderungen der Hygiene bei Operationen und anderen invasiven Eingriffen. Bundesgesundheitsblatt - Gesundheitsforschung - Gesundheitsschutz 2000; 43: $644-648$

6 Robert Koch-Institut. Vorwort und Einleitung der Kommission zur Richtlinie für Krankenhaushygiene und Infektionsprävention. Bundesgesundheitsblatt - Gesundheitsforschung - Gesundheitsschutz 2004; 47: 409-411

7 Anonym. Technische Regeln für Biologische Arbeitsstoffe 001: Allgemeines und Aufbau des technischen Regelwerks zur Biostoffverordnung - Anwendung von Technischen Regeln für Biologische Arbeitsstoffe - TRBA (Ausgabe Mai 2000) www.baua.de $>$ Themen von A-Z > Biologische Arbeitsstoffe $>$ Technische Regeln für Biologische Arbeitsstoffe (TRBA)

8 Schulze-Röbbecke R. Was ist Hygiene? Krankenhaushygiene up2date 2007; 2: 1 -4 